\title{
Caracterización epidemiológica y clínica de las cinco dermatosis más frecuentes en consulta externa pediátrica dermatológica
}

\author{
Clinical and epidemiological characterization of the five most common skin diseases \\ in the dermatologic practice
}

\section{José Gamaliel Ferrufino Euceda, * Héctor Rubén Caballero Castro, ** Juan José Guerra,***}

\section{RESUMEN}

Antecedentes: Las dermatosis son patologías que se presentan frecuentemente en poblaciones de países en vías de desarrollo. Las enfermedades de la piel constituyen uno de los principales motivos de consulta externa general, representan del 6 al 24\% de la consulta pediátrica. Objetivo: Caracterizar epidemiológica y clínicamente las cinco dermatosis frecuentes en consulta dermatológica pediátrica del Instituto Hondureño de Seguridad Social, Región Noroccidental (IHSS-HRN) en el período de mayo a septiembre de 2015. Pacientes y Métodos: Estudio cuantitativo, descriptivo, transversal, realizado en niños de 0 meses a 11 años que reunieron los criterios para participar en el estudio de las cinco dermatosis más frecuentes. Los datos se recolectaron mediante una encuesta aplicada al responsable o tutor del niño participante. Resultados: Las primeras 5 dermatosis en frecuencia fueron: Dermatitis atópica 133 (40\%), prurigo por insectos 58(18\%), molusco contagioso 56 (17\%), urticaria 44 (13\%) impétigo 38 (12\%), Los 329 pacientes evaluados provenían del casco urbano. Todos pertenecían a la etnia mestiza, a excepción de un afro descendiente. El método diagnóstico fue clínico en el $100 \%$ de los casos. Conclusiones: Las dermatosis inflamatorias no infecciosas son la causa de problemas dermatológicos en niños probablemente debido al cambio de hábitos alimenticios, exposiciónambiental y componente genético.

* Médico residente de tercer año del posgrado de Pediatría. Universidad Nacional Autónoma de Honduras, Valle de Sula, UNAH-VS.

** Dermatólogo Pediatra, Instituto Hondureño de Seguridad Social. I.H.S.S.

*** Master en Salud Pública, UNAH-VS.

Dirigir correspondencia a: gamaferrufino@hotmail.com

Recibido: 15 de noviembre 2015 Aprobado: 12 de enero 2016

\section{PALABRAS CLAVE}

Dermatitis, Enfermedades de la piel, Niño.

\section{ABSTRACT}

Background: skin diseases frequently occur in children in developing countries and they represent 6 to 24\% of pediatric consultation. Objective: To characterize most frequent skin diseases in the pediatric dermatologic practice at the Northwestern Regional Hospital-Honduran Social Security Institute (IHSS-HRN) in the period between May and September 2015. Patients and Methods: This is a quantitative, descriptive, cross-sectional study conducted in children from 0 months to 11 years who met the criteria for participation in the study of being diagnosed with the five most common skin diseases. Data was collected through a survey applied to the participating child or their guardian. Results: Children were diagnosed with atopic Dermatitis 133 (40\%), prurigo due to insect bites 58 (18\%), molluscum contagiosum 56 (17\%), urticaria 44 (13\%) and impetigo 38 (12\%). 329 patients lived in an urban setting. Except for an afro-descendant, all of them belong to a mixed ethnicity. The diagnosis was clinical method in $100 \%$ of cases. Conclusions: Non-infectious inflammatory skin diseases are the cause of skin problems in children probably due to changes in eating habits, environmental exposure and genetic component.

\section{KEYWORDS}

Dermatitis, Skin diseases, child.

\section{INTRODUCCION}

Las dermatosis son patologías que se presentan frecuentemente en poblaciones de países 
en vías de desarrollo. Constituyen uno de los principales motivos de consulta externa general y representan del 6 al $24 \%$ de la consulta pediátrica. ${ }^{(1,2)}$ Hay pocos estudios en países en vías de desarrollo que le den la importancia a estas enfermedades, las cuales suponen un gran problema de salud pública. Esta conducta se debe a que las enfermedades cutáneas raras veces ponen en peligro la vida de los paciente y por lo tanto no han sido consideradas lo suficiente como para prestarles la debida atención. ${ }^{(3)}$

Las patologías dermatológicas dependen de diferentes factores sociales, económicos, nutricionales, ambientales y climáticos, así como también a los factores genéticos particulares que cada población presenta. ${ }^{(2)}$

Algunas dermatosis son compartidas por poblaciones menores de 5 años de Latinoamérica especialmente Centroamérica y países de Sudamérica, al igual que en África, tales como las infecciosas (verrugas e impétigo) dermatitis atópica, infestaciones parasitarias (escabiosis y pediculosis) y las relacionadas con el medio ambiente (pitiriasis alba).(4) En los países desarrollados tales como Gran Bretaña, Alemania, Canadá y Estados Unidos de Norteamérica la dermatitis atópica es la más frecuente, representando un 25 a 33\% del total de las consultas, seguida de los nevos melanocíticos, con cifras que oscilan entre el 3 y $20 \%$, o las verrugas que representan alrededor del 5-13\%. Los resultados son diferentes en países en vías de desarrollo, en los que predominan las infecciones y las infestaciones. ${ }^{(5)}$

Las dermatosis más frecuentes en la población escolar en los países en vías de desarrollo, según la OMS son pioderma (impétigo, foliculitis, forúnculo, ántrax), infecciones virales (verrugas, molusco contagioso), escabiosis y otras infecciones por ectoparásitos (pediculosis capitis, p. corporis), tiña capitis y otras micosis superficiales (dermatofitosis, pitiriasis versicolor) y las dermatitis (atópica, por contacto). ${ }^{(6)}$
En Honduras no se cuenta con estudios documentados de prevalencia de dermatosis más frecuentes en población infantil. El objetivo de este estudio es Caracterizar epidemiológica y clínicamente las cinco dermatosis más frecuentes en consulta externa pediátrica dermatológica del Instituto Hondureño de Seguridad Social, Región Noroccidental en el período de mayo a septiembre de 2015.

\section{PACIENTES Y METODOS}

Se realizó un estudio cuantitativo, descriptivo, transversal en la consulta externa pediátrica dermatológica del Instituto Hondureño de Seguridad Social-Hospital Regional del Norte (IHSS-HRN) en el período comprendido entre mayo a septiembre del 2015. El universo fueron los 507 niños de 0 a 11 años de edad (establecidos por la Institución como edad pediátrica) atendidos en dicho periodo. La muestra fueron los 329 pacientes que presentaron las cinco dermatosis más frecuentes. Se excluyeron los pacientes que no tenían el diagnóstico identificado dentro de las cinco dermatosis más frecuentes y los pacientes a los que sus tutores legales no aceptaron el consentimiento informado.

Todos los datos se obtuvieron mediante un Instrumento tipo formulario conteniendo variables sociodemográficas (edad, sexo, procedencia y etnia), forma clínica (inflamatoria no infecciosa, infecciosa viral e infecciosa bacteriana) y diagnóstico clínico o laboratorial. Dicho formulario, fue llenado por el médico tratante en la consulta externa de dermatología pediátrica, con datos proporcionados por el padre o mentor del niño participante.

Para el análisis estadístico se hizo uso del cálculo de frecuencia, porcentajes y se utilizó el paquete de Microsoft EXCEL 2013 y EPI INFO Versión 6 para la tabulación y presentación final del estudio.

Aspectos éticos: este estudio fue sometido a dictamen del Comité de Ética en Investigación 
Biomédica del Instituto Hondureño de Seguridad Social-Hospital Regional del Norte/Universidad Autónoma de Honduras, se solicitó el consentimiento informado por parte de los padres/madres/ tutores.

\section{RESULTADOS}

Según la distribución por edad de las 5 dermatosis más frecuentes en consulta externa pediátrica se dividió en; lactantes 134 (41\%), preescolar $188(57 \%)$ y escolares 7 (2\%). En cuanto a sexo, se encontró la siguiente distribución; femenino 179 casos (55\%) y masculino 150 casos (45\%).

Entre las cinco dermatosis más frecuentes, se encontraron: Dermatitis atópica $133(40 \%)$ y prurigo por insectos 58 (18\%).

(Ver Gráfica No.1)

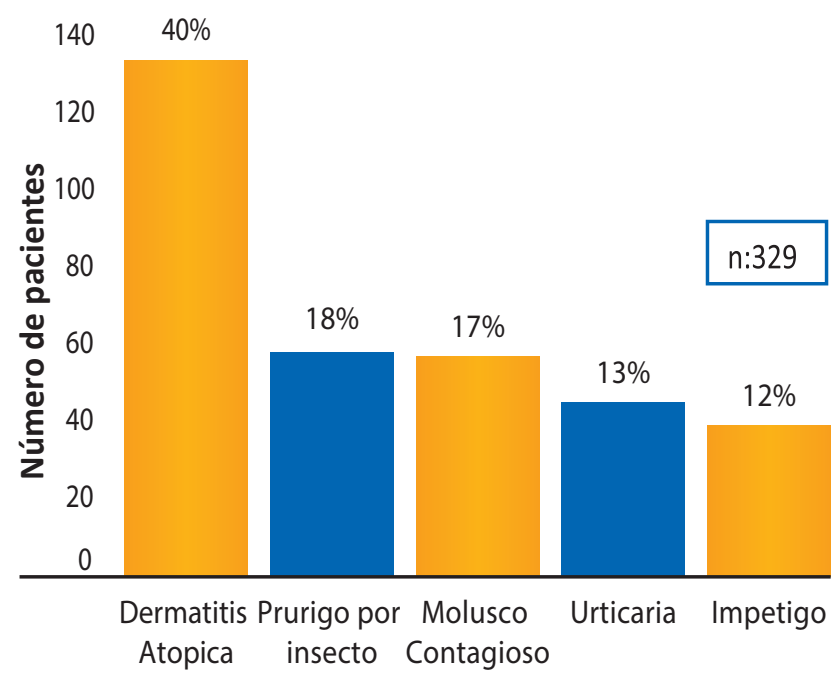

\section{Gráfica No. 1: Dermatosis frecuentes en la consulta externa de Dermatología IHSS}

Fuente: Investigación Dermatosis más frecuentes en Pediatría en IHSS-HRN, 2015

En cuanto a la distribución del tipo de dermatosis más frecuente respecto al grupo etario se encontró que: 83 (62.5\%) de las dermatitis atópica, se presentaron en el grupo de los lactantes y 49 (84.5\%) de los casos de prurigo por insectos se presentaron en el grupo de los preescolares. (Ver Tabla No. 1)
Tabla No.1: Distribución de Dermatosis más frecuentes por Grupo Etario

\begin{tabular}{|c|c|c|c|c|}
\hline Dermatosis & Lactantes & Preescolare & 5 Escolares & $\mathbf{n}$ \\
\hline $\begin{array}{l}\text { Dermatitis } \\
\text { atópica }\end{array}$ & $83(62.5)$ & $48(36 \%)$ & $2(1.5 \%)$ & $\begin{array}{l}133 \\
(100 \%)\end{array}$ \\
\hline $\begin{array}{l}\text { Prurigo por } \\
\text { insecto }\end{array}$ & $8(14 \%)$ & 49 (84.5\%) & $1(1.5 \%)$ & $\begin{array}{l}58 \\
(100 \%)\end{array}$ \\
\hline $\begin{array}{l}\text { Molusco } \\
\text { contagioso }\end{array}$ & $24(43 \%)$ & 32 (57\%) & $0(0 \%)$ & $\begin{array}{l}56 \\
(100 \%)\end{array}$ \\
\hline Urticaria & $6(14 \%)$ & 34 (77\%) & $4(9 \%)$ & $\begin{array}{l}44 \\
(100 \%)\end{array}$ \\
\hline Impetigo & $13(34 \%)$ & 25 (66\%) & $0(0 \%)$ & $\begin{array}{l}38 \\
(100 \%)\end{array}$ \\
\hline Total & 134 & 188 & 7 & $\begin{array}{l}329 \\
(100 \%)\end{array}$ \\
\hline
\end{tabular}

Fuente: Investigación Dermatosis más frecuentes en Pediatría en IHSS-HRN, 2015

Al clasificar las dermatosis por sexo, se encontró que la infección por molusco contagioso estaba presente en pacientes del sexo femenino en 34 casos (61\%) versus 22 casos $(49 \%)$ del masculino, además se encontró que el $52 \%$ de los casos de urticaria se presentó en pacientes del sexo masculino. (Ver Tabla No. 2)

Tabla No. 2: Distribución de Dermatosis por Sexo

\begin{tabular}{llll}
\hline Dermatosis & \multicolumn{3}{l}{ Femenino } \\
\hline Dermatitis atópica & $72(55 \%)$ & $61(45 \%)$ & $133(100 \%)$ \\
Prurigo por insecto & $31(53.5 \%)$ & $27(46.5 \%)$ & $58(100 \%)$ \\
Molusco contagioso & $34(61 \%)$ & $22(39 \%)$ & $56(100 \%)$ \\
Urticaria & $21(48 \%)$ & $23(52 \%)$ & $44(100 \%)$ \\
Impetigo & $21(55 \%)$ & $17(45 \%)$ & $38(100 \%)$ \\
Total & 179 & 150 & 329 \\
\hline
\end{tabular}

Fuente: Investigación Dermatosis más frecuentes en Pediatría en IHSS-HRN, 2015

Al clasificar las dermatosis en inflamatorias no infecciosas e infecciosas, se encontró que 235 (71\%) eran inflamatorias no infecciosas.

(Ver Tabla No. 3) 


\section{Tabla No. 3: Presentación Clínica de las dermatosis más frecuentes}

\begin{tabular}{lcc}
\hline Presentación Clínica de Dermatosis & $\mathbf{n = 3 2 9}$ & Porcentaje \\
\hline $\begin{array}{l}\text { Inflamatorias no infecciosas } \\
\text { (dermatitis atópica, prurigo } \\
\text { por insectos y urticaria) }\end{array}$ & 235 & $71 \%$ \\
Viral (molusco contagioso) & 56 & $17 \%$ \\
Bacteriana (Impétigo) & 38 & $12 \%$ \\
\hline
\end{tabular}

Fuente: Investigación Dermatosis más frecuentes en Pediatría en IHSS-HRN, 2015

\section{DISCUSIÓN}

Debido al advenimiento de la industrialización en países en vías de desarrollo, así como cambios en la dieta del infante y componentes genéticos, las dermatosis infamatorias no infecciosas son cada vez más frecuentes. La dermatitis atópica ha tomado realce afectando al $2 \%$ y $5 \%$ de la población general y entre el 10-20\% en la población infantil. Las cifras son más altas en las zonas urbanas y en las poblaciones con mayor nivel socioeconómico. Presenta su pico de incidencia en menores de 2 años, sin embargo, esta se puede extender hasta la edad escolar con antecedentes de piel seca y atopias. ${ }^{(7,8,)}$

En este estudio se evidenció que la primera causa de consulta dermatológica en consulta externa fue la dermatitis atópica en un $40 \%$ de los pacientes, especialmente en los lactantes (62\%). Esto se debe a la vulnerabilidad en cambios alimenticios, uso de ropa de lana, aunado al no cumplimiento de los padres a las recomendaciones para la prevención de esta enfermedad.

Las picaduras por insectos son muy comunes en los niños debido a su exposición al aire libre, sobre todo en países con clima tropical. La exposición a los insectos afecta cualquier área corporal, se presentan vesículas en la fase inicial posteriormente aparecen pequeñas pápulas eritematosas. Las lesiones son muy pruriginosas, se producen costras hemáticas secundarias al rascado por lo que es un motivo frecuente de consulta dermatológica, afecta sobre todos a niños preescolares entre 2 a 5 años, no existe predominancia por género o etnia. ${ }^{(9,10)}$ Lo que concuerda con los resultados de este estudio, donde se demostró que los pacientes afectados fueron los preescolares $84.5 \%$, esto debido a mayor exposición por su edad a la práctica de juegos recreativos y al uso de ropa no adecuada. Todos los niños proceden de la zona norte sumamente tropical, tampoco hubo predominancia significativa por sexo ( $53 \%$ en pacientes femeninos) en estos niños.

La urticaria en su presentación aguda en población infantil representa entre el 10 al $20 \%$ de las consulta dermatológica en países desarrollados como España, Gran Bretaña, Canadá y Estados Unidos, y cada vez más frecuente en países en vías de desarrollo. Se define como un síndrome reactivo de piel y mucosas caracterizado por edema y ronchas pruriginosas, puede presentarse a cualquier edad. La urticaria tiene un carácter multifactorial con componente inmunitario, no hay predominio de sexo o etnia. ${ }^{(11,12)}$ En este estudio, los resultados concuerdan con lo reportado en la literatura, pues se encontró que 13\% de la consulta dermatológica más frecuente es debido a urticaria, sin embargo fue más frecuente en los preescolares $77 \%$, probablemente debido a la incorporación de alimentos más alergénicos en la dieta luego de la ablactación, además se encontró que la diferencia en cuanto al sexo no fue significativa (masculino $52 \%)$.

Las dermatosis de tipo viral en países desarrollados son la tercera causa de la consulta externa dermatológica representan un $5-13 \%$ de incidencia de casos, el molusco contagioso es una infección cutánea altamente contagiosa, caracterizada por pápulas hemisféricas, de superficie lisa, umbilicadas de crecimiento lento. Es una enfermedad rara antes de primer año. Su incidencia máxima se da a los 2-5 años. 
Es frecuente en zonas cálidas sin predominio de sexo o etnia. ${ }^{(13)}$ En este estudio se encontró que fue la tercera causa de consulta externa dermatológica $17 \%$, presentándose con mayor frecuencia en los preescolares $57 \%$, probablemente porque llevan menos ropas y están en mayor contacto con otros niños u objetos contaminados, cabe destacar que a diferencia de lo que reporta la literatura, en este estudio se encontró predominio en el sexo femenino $61 \%$.

Las dermatosis de tipo bacteriano según la OMS representa en países en vías de desarrollo, la principal causa de consulta dermatológica en población en edad escolar, debido a que el hacinamiento y el escaso acceso a recursos básicos facilita la propagación de dichas enfermedades. El impétigo es extremadamente frecuente, definido como una infección cutánea superficial causada por Streptococcus pyogenes, Staphylococcus aureus o ambos, se presenta sobre todos en niños mayores de 2 años sin predilección por sexo. ${ }^{(14,15)}$ En discordancia con los hallazgos de este estudio, pues aunque el impétigo estaba entre las dermatosis más frecuentes, ocupó el quinto lugar con $12 \%$, probablemente debido al tipo de pacientes que se atienden en el IHSS, y que el $100 \%$ eran procedentes del área urbana.

En conclusión, fueron las dermatosis inflamatorias no infecciosas las más frecuentes en este estudio específicamente la dermatitis atópica, como en los países industrializados, probablemente debido al cambio de hábitos alimenticios, exposición ambiental y componente genético.

\section{BIBLIOGRAFÍA}

1. Uriarte J, Sáez de Ocariz M, Durán McKinster C, Orozco Covarrubias L, González R, Ruiz Maldonado R. Variación estacional de las dermatosis más frecuentes en una consulta externa de Dermatología Pediátrica en México. Dermatol Pediatr Lat. 2005; 3(1): 21-25.

2. Katsarou A, Armenaka M, Maria Kosmadaki, Eirini Lagogianni, Vasiliki Vosynioti, Andreas Katsambas. et al. Skin diseases in Greek and immigrant children in Athens. Int J Dermatol 2012; 51:173-177.

3. Discussion papers in Child Health. Epidemiology and management of common skin diseases in children in developing countries. WHO/FCH/CAH/05.12.

4. Del Pozzo BR, Lazo Langner A, Gutiérrez Castrellón P, Ruiz Maldonado R, Dermatosis comunes en niños mexicanos: estudio comparativo entre dos décadas. Rev ISRN Dermatology. 2012;1(12):1-5.
5. Casanova JM, Sanmartín V, Soria X. Dermatosis infantiles en la consulta de Dermatología de un hospital general universitario en España. Actas Dermosifiliogr. 2008; 99:111-118.

6. Sellarés Casas E, Moraga A. Infecciones cutáneas bacterianas. Revista de la Asociación Española de pediatría [Revista en internet] 2006. [Citado 14 de junio del 2013]; 25-39 Disponible en: http:// www.aeped.es/sites/default/files/docu mentos/infeccionescutaneas

7. Rosalía Ballona Chambergo. Update in Pediatric Dermatology. Rev. Perú. pediatr. 2006; 1 (2).

8. Llorente Galván $M$, Lequerica Segrera $P$, Álvarez Pereira E, Dermatitis atópica: enfoque clínico y terapéutico básico. Rev Cienc biomed. 2010.1 (2): 271-77.

9. Klotz JH, Klotz SA, Pinnas JL. Animal Bites 
and Stings with Anaphylactic Potential. JEM. 2009; 36(2): 148-56.

10. Beirana A. Prurigo por Insectos. PAC Dermatología. Sociedad Mexicana de Dermatología: Dermatología Pediátrica. Ed.1era, № 5:29-30.2010.

11. Tincopa-Wong OW. Urticaria y angioedema: una visión general. Acta Med Per. 2014: 31 (2):111-124.

12. Guillén Escalón J, Rosas Vargas MA, Mendoza Magaña E, Zepeda Ortega B, Sienra Monge JJL, del Río Navarro BE. Urticaria y angioedema. Revista Alergia México 2007;54(2):54-65.
13. Salvador Herrero LA, Sidro Bou, Luisa $F$, Pérez Cervelló G, Freixenet Guitart N, Balanza Garzón A, et al. Molluscum contagiosum. Guías clínicas 2007; 7 (22).

14. Guillén Fiel G, Santos García M, Ureta Velasco N, Rojo Conejo P. Departamento de Pediatría. Hospital General Universitario «12 de Octubre». Madrid. Tratamiento de impétigo. Acta Pediatr Esp. 2008; 66(8): 415-417.

15 Sellarés Casas E, Moraga A. Infecciones cutáneas bacterianas. Revista de la Asociación Española de pediatría [Revista en internet] 2006. [Citado 14 de junio del 2013]; Disponible en: http://www.aeped. es/sites/default/files/documentos/infeccio nescutaneas. 